\title{
1092 Channel 2-D Array Demultiplexer for Ultralarge Data Bandwidth
}

\author{
Trevor K. Chan, Jason Karp, Rui Jiang, Nikola Alic, Stojan Radic, Christopher F. Marki, and Joseph E. Ford
}

\begin{abstract}
We demonstrate $1 \times 1092$ channel wavelength demultiplexing with 50-GHz channel pitch and a 600-nm total bandwidth. Outputs from $1 \times \mathbf{4 0}$ channel arrayed waveguide gratings operating with multiple orders enter a free-space optical grating demultiplexer which separates the orders into a 2-D spot array, where the light can be coupled into discrete output fibers or operated on by a surface normal device (i.e., microelectromechanical system switch or detector array). Supercontinuum source input from 1140 to $1750 \mathrm{~nm}$ produced a $28 \times 39$ spot array at the output plane. The insertion loss for light is coupled into a single mode fiber ranged from 7 to $18 \mathrm{~dB}$ with less than 10-dB loss in channels between 1300 and $1750 \mathrm{~nm}$. Bit-error-rate measurements show a negligible $0.1-\mathrm{dB}$ power penalty at $10 \mathrm{~GB} / \mathrm{s}$.
\end{abstract}

Index Terms-Broadband communication, gratings, microelectromechanical systems (MEMS), optical communication, wavelength division multiplexing.

\section{INTRODUCTION}

$\mathbf{T}$ HE POTENTIAL bandwidth capacity of optical fibers, estimated to be over $100 \mathrm{~Tb} / \mathrm{s}$ [1], remains unfulfilled in modern fiber communication systems, which reach up to $1.6 \mathrm{~Tb} / \mathrm{s}$. Even in research experiments, the maximum data capacity shown is on the order of $12 \mathrm{~Tb} / \mathrm{s}$ [2]. Achieving $100-\mathrm{Tb} / \mathrm{s}$ capacity requires a combination of large channel counts and large per-channel data rates, with nearly an order of magnitude increase in wavelength spectrum over the $\sim 100 \mathrm{~nm}$ used in current communication systems. One key component in reaching this goal is a large channel count and broad spectralbandwidth wavelength multiplexer to combine and separate the data streams. Wavelength multiplexers are also instrumental in other wavelength management components including channel level monitors, wavelength switches, and spectral power equalizers.

Commercial wavelength multiplexers, including both waveguide and planar grating-based components, use a single diffractive element to separate wavelength channels into a spatially linear 1-D array of outputs. This is practical when dealing with approximately 100 channels, but a single linear array becomes problematic for large channel counts. For example, the linear alignment of 1000 collection fibers at

Manuscript received January 5, 2006; revised November 10, 2006. This work was supported in part by the Natural Science and Engineering Research Council of Canada.

The authors are with the Department of Electrical and Computer Engineering, University of California, San Diego, La Jolla, CA 92093 USA (e-mail: tkc@ucsd.edu; jkarp@ucsd.edu; rjiang@ucsd.edu; nalic@ucsd.edu; radic@ece.ucsd.edu; cmarki@ucsd.edu; jeford@ucsd.edu).

Color versions of one or more of the figures in this paper are available online at http://ieeexplore.ieee.org.

Digital Object Identifier 10.1109/JLT.2006.889686

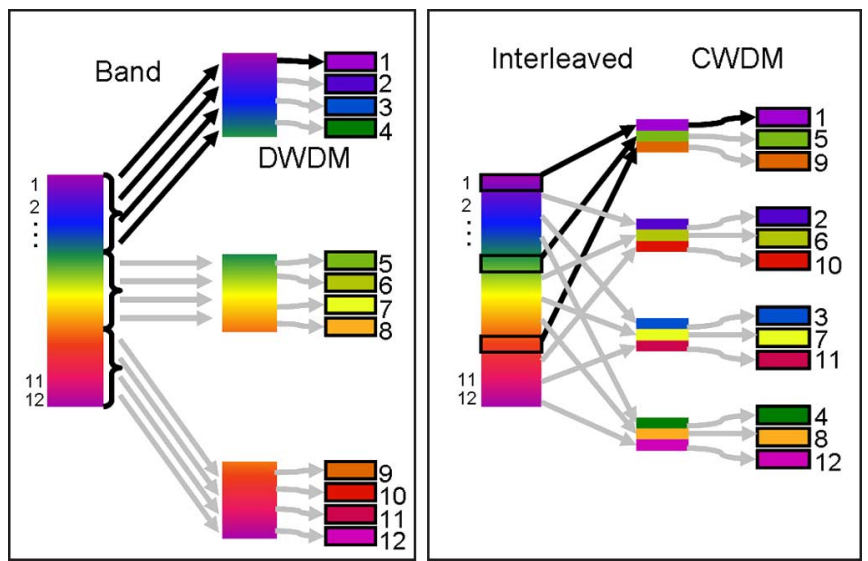

Fig. 1. Two approaches to hierarchical wavelength multiplexing. (Left) Banded. (Right) Interleaved.

a $125-\mu \mathrm{m}$ pitch (from the diameter of single mode fiber) requires submicrometer alignment accuracy for each fiber in a $125-\mathrm{mm}$-long array. This is a major packaging challenge especially for components which may need to be hermetically sealed, leading to costly and potentially unreliable components.

This problem is avoided by using hierarchical (multistage) multiplexing. Takada et al. showed multistage demultiplexing using a cascade of two arrayed waveguide gratings (AWG) [3]. A course-spectral-pitch multiplexer was followed by multiple fine spectral multiplexers. The first stage consisted of a single AWG to create 25 channels spaced by $1 \mathrm{THz}(8 \mathrm{~nm})$. Each of these channels was further divided into 168 channels spaced at $5 \mathrm{GHz}(0.04 \mathrm{~nm})$ by the second stage of AWG. A separate AWG is used for each output of the primary stage, leading to 26 individually packaged components. The entire multiplexer produced a total of 4200 channels over a $159-\mathrm{nm}$ spectrum. Assuming $80 \%$ bandwidth utilization, reasonable for the AWG Gaussian passbands, Takada's staged demultiplexer potentially allows up to 16.8-THz aggregate data bandwidth.

There are two categories of multistage multiplexing, as shown in Fig. 1. "Banded" multiplexers, as a first stage, use a course-pitch filter to separate spectrally adjacent clusters of channels, and as a second stage, a set of fine-pitch filters which further separate these groups into individual channels. Takada's is one example of a banded multiplexer. The alternative "interleaved" multiplexers, as a first stage, use a filter which isolates sets of individual data channels separated by some multiple of the individual channel pitch. The second stage is a relatively course filter which further separates these widely spaced channels into individual output channels. More complex (higher level) hierarchical multiplexers can also be constructed 


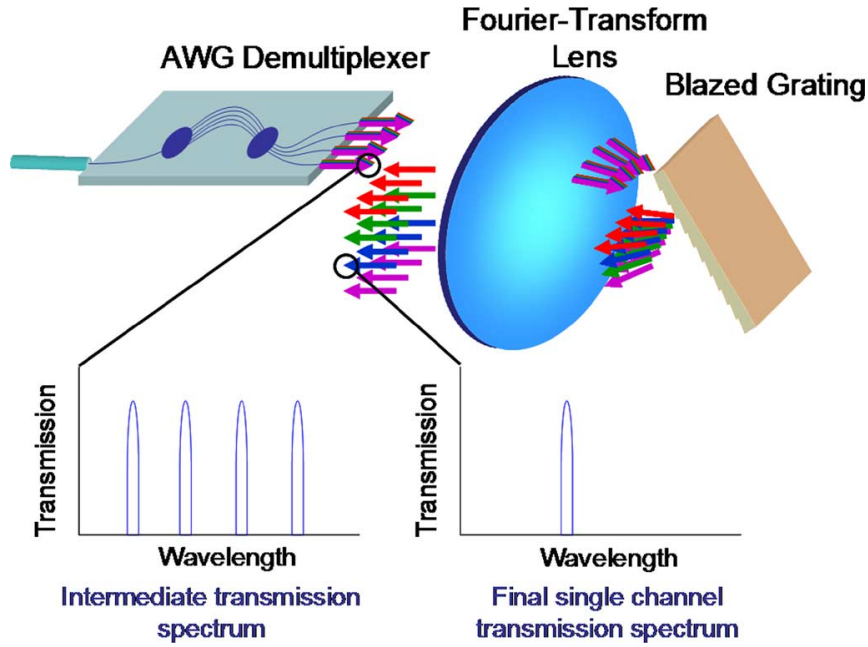

Fig. 2. Hybrid waveguide-FSG multiplexer producing a 2-D array output.

with three or more stages, potentially combining both banded and interleaved approaches.

AWG multiplexers are normally operated with a restricted spectral bandwidth so that each output waveguide carries a single channel. This is true in the multiplexer of Takada et al. [3] as each secondary AWG receives a signal that has been filtered to within one free spectral range (FSR) by the first AWG stage, which has a much wider FSR. However, as with all diffractive elements, AWG can yield multiple diffraction orders for a sufficiently broad input spectrum.

An interleaved multiplexer can be constructed using, as the first stage, an AWG in this higher order mode, where the input spectrum is much larger than the FSR. Each output waveguide then carries multiple channels, each corresponding to one diffraction order, and each separated from the adjacent channels by the AWG's FSR. These channels can be separated into individual waveguides by the second stage. Because the firststage output produces fine channels with large gaps, the secondstage multiplexer can be a course wavelength multiplexer with narrow passbands. Free-space-grating (FSG) multiplexers are ideal for the second stage, especially because a single optical multiplexer can operate with a row of parallel inputs, provided the direction of dispersion is oriented perpendicular to the input row, so that the final output is a 2-D array of individual wavelength channels. The resulting system is shown in Fig. 2.

Such a hybrid multiplexer was proposed by Dragone and Ford in 2001 [4]. In 2004, Weiner and Xiao successfully demonstrated a related array multiplexer using a virtual imaged phased array multiplexer operated with multiple diffraction orders, followed by an FSG demultiplexer to create a 2-D array output [5]. This system produced 41 demultiplexed channels (four rows of ten channels) with about $1.75-\mathrm{GHz}$ spectral bandwidth per channel, yielding a potential aggregate data bandwidth of $71.75 \mathrm{GHz}$. In 2005, we demonstrated an array multiplexer using the system in Fig. 2, a $1 \times 8$ AWG with 50-GHz pitch, and an FSG second stage, yielding 72 demultiplexed channels (nine rows of eight channels), which, again assuming a maximum spectral channel utilization of $80 \%$, provided a potential aggregate data capacity of $2.88 \mathrm{~Tb} / \mathrm{s}[6]$.

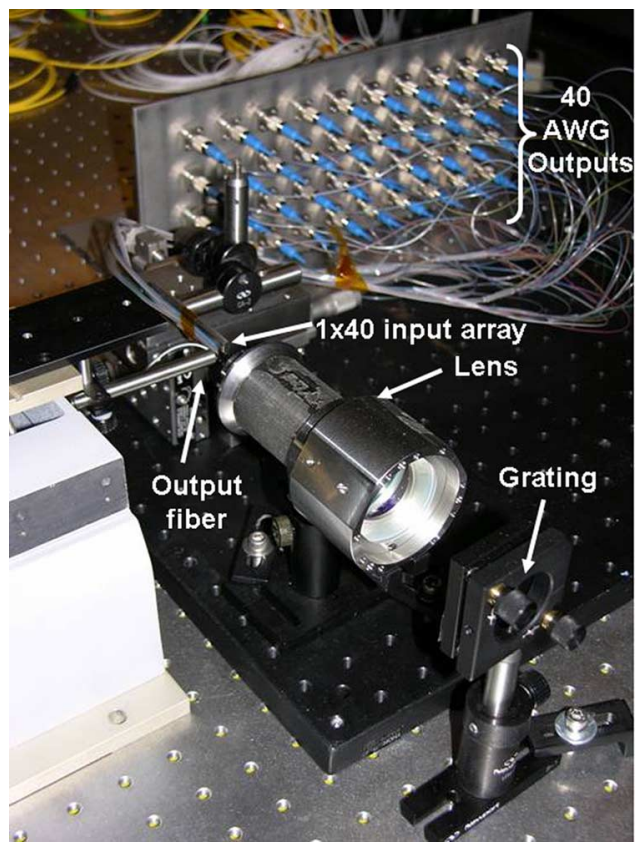

Fig. 3. Experimental setup showing the v-groove array that carries the 40 outputs from the first-stage multiplexer and the FSG multiplexer that produces a 2-D output array which is tested using a single individually aligned output fiber for channel characterization.

In this paper, we extend our earlier results to an ultralarge data capacity multiplexer which carries over 1000 individual channels at approximately $50-\mathrm{GHz}$ pitch and spreads over a 600-nm wavelength spectrum for an aggregate potential data capacity of over $40 \mathrm{~Tb} / \mathrm{s}$. We characterize the performance of individual demultiplexed channels using a single-mode output fiber. This optical system, however, is primarily intended to be integrated with optoelectronic or micromechanical devices to construct wavelength switches and dynamic spectral equalizers for ultralarge data capacity networks.

\section{DemultipleXer Setup}

Fig. 3 shows a photograph of the experimental system. The first stage of our system consisted of a conventional $1 \times 40$ AWG demultiplexer with 50-GHz-output channel pitch. The 40th channel of the AWG had anomalously high loss, and therefore, only 39 outputs were used. At $1560 \mathrm{~nm}$, the AWG showed less than 5-dB insertion loss and a 27.2-nm FSR. The relation between FSR, center wavelength $\lambda_{c}$, and diffraction order $m$ is given by [7]

$$
\mathrm{FSR}=\lambda_{c}\left[\frac{m}{m-1}-1\right] .
$$

We find that the channels near $1560 \mathrm{~nm}$ lie in the 53rd diffraction order. The AWG is designed for this order and is typically not used outside of this range. However, other diffraction orders are produced with equally high transmission efficiency when the AWG is illuminated outside of this range.

For our system, we illuminated the AWG with a high brightness supercontinuum source with spectral output ranging 


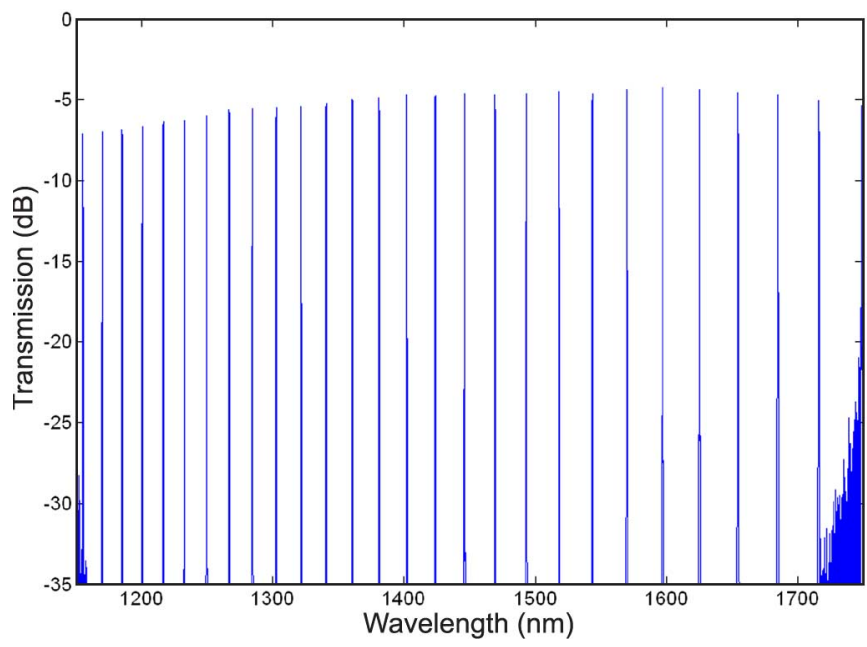

Fig. 4. Transmission spectra carried by a single-AWG output fiber when illuminated with a $600-\mathrm{nm}$ bandwidth showing 28 diffraction orders separated by approximately $25-\mathrm{nm}$ FSR. This is the first-stage (partially demultipled) output of our demultiplexer.

from 1150 to well over $1800 \mathrm{~nm}$. With this source, the AWG operation bandwidth was limited only by the spectral range of our optical spectrum analyzers (OSAs) from 1100 to $1800 \mathrm{~nm}$. With a 600-nm input bandwidth, the AWG produces a spectral comb of at least 28 diffraction orders in each output channel, as shown in Fig. 4.

All outputs of the AWG must be integrated with the secondstage (FSG) multiplexer to create a fully demultiplexed output. Ideally, the AWG waveguide would be directly integrated with the second stage, as shown in Fig. 2, avoiding intermediate fiber coupling losses. In our system, however, we connected a fully packaged AWG to a $1 \times 40$ linear v-groove array with polished fibers at a $127-\mu \mathrm{m}$ pitch.

The second-stage demultiplexer consists of a $5-\mathrm{cm}$ focallength Fourier transform lens followed by a $75-1 \mathrm{p} / \mathrm{mm}$ blazed reflection grating. The grating is coated with aluminum for adequate reflectivity of a broad spectrum of visible-to-near infrared wavelengths. The grating is oriented near Littrow angle, so that the first-order diffracted output is backreflected and returns through the Fourier lens and is refocused, creating an image of the v-groove array adjacent to itself. Since the grating is a diffractive element, the horizontal position of the image is determined by its wavelength. Since each diffraction order lies at a different wavelength, we generate multiple images which are horizontally displaced from each other. Moreover, the image of the array appears slanted due to wavelength variations among the channels within a diffraction order. We tilt the v-groove array in the opposite direction to align the spots in a vertical column. With a broadband input into our system, this ultimately produces a rectangular array of spots raster positioned according to their wavelength. Each of these spots represents the output position of a demultiplexed wavelength channel. The signal coupled to an optical fiber positioned at one of these output spots is shown in Fig. 5, showing suppression of all, but one diffracted order is visible in Fig. 4. This is the fully demultiplexed (single channel) output of the hybrid demultiplexer.

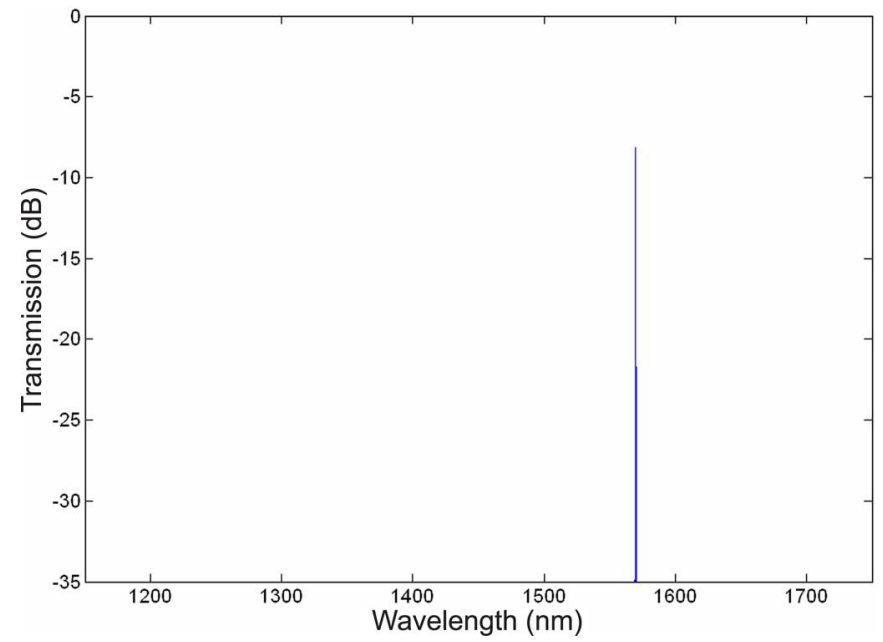

Fig. 5. Transmission spectra coupled to a fiber positioned at the image plane of the second-stage FSG demultiplexer showing a single-AWG diffraction order in the final output.

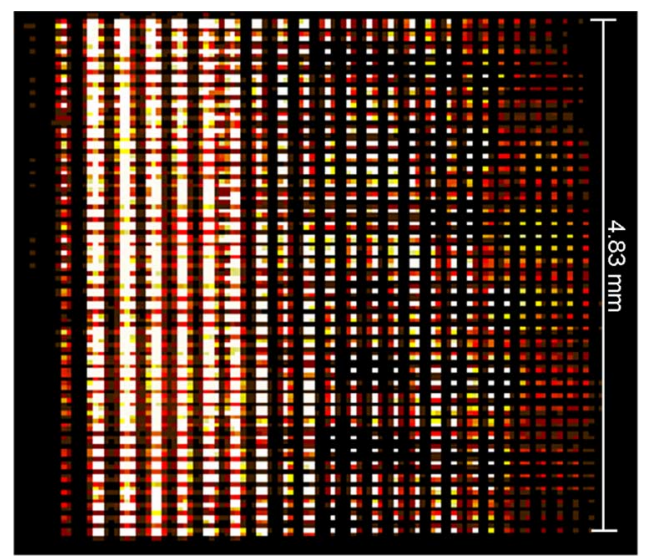

Fig. 6. Photograph of the entire output array taken by relay imaging the output into an InGaAs sensor array showing the 39 columns (first-stage AWG fiber inputs) of 28 diffraction orders each (dispersed by the FSG). The $320 \times 240$ InGaAs camera does not fully resolve the 1090 spots.

\section{ARrAy-DemultipleXer OUtPut}

We photographed the entire spot array using an infrared camera, a Sensors Unlimited 320 M camera with a $320 \times 240$ array of InGaAs detector pixels at a $25-\mu \mathrm{m}$ pitch. The output array is located adjacent to the fiber $\mathrm{v}$-groove array; therefore, to obtain this photograph, we temporarily inserted a gold mirror to reflect the light out of the demultiplexer and into an imaging system consisting of two identical Fourier transform lenses in a unit-magnification $4 \mathrm{f}$ configuration. The image of the spot array is shown in Fig. 6. Each column of the array represents a single-AWG diffraction order, or in other words, a single image of the intermediate v-groove array. These spots are raster positioned according to wavelength from top to bottom and then from left to right. The nonuniformity of the array is a result of several factors, including the relatively course sampling grid of the detector pixels, the nonuniformity of the supercontinuum source (which varied by $15 \mathrm{~dB}$ over the $600-\mathrm{nm}$ operating range), and wavelength dependent losses. An accurate measure of the insertion loss for each wavelength channel is shown in the following section. 


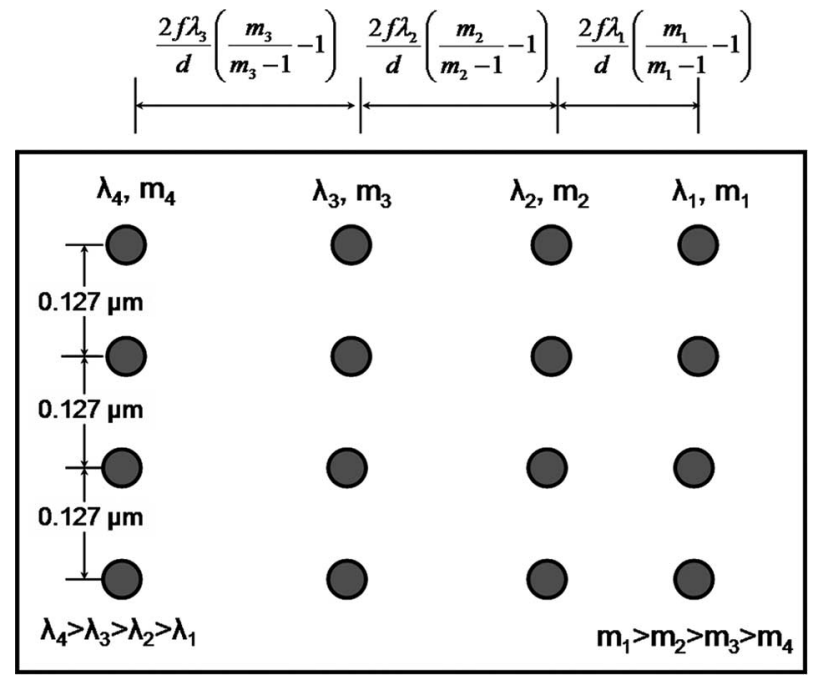

Fig. 7. Expected arrangement of the output spots. The vertical separation of the rows is constant, assuming negligible imaging system distortion, but the space between columns is a function of the diffraction order.

A standard SMF-28 single mode fiber mounted in a silicon $\mathrm{v}$-groove and polished flat was used to characterize each of the 1092 individual channel outputs. The fiber was mounted on a PI model F206 hexapod stage (visible at left in Fig. 3) to provide $<0.1-\mu \mathrm{m}$ absolute positioning accuracy. This fiber was scanned across the plane of best overall focus. The fiber coupled signal was connected to the external components for intensity measurements, spectral analysis, polarization dependence loss measurements, and bit-error-rate (BER) tests.

Fig. 7 diagrams the calculated output-spot distribution. The rows are separated by $0.127 \mathrm{~mm}$, which is equal to the pitch of the $1 \times 40 \mathrm{v}$-groove array. The separation between the columns is set by the dispersion from diffraction from the FSG, and so is proportional to the FSR (1) and increases monotonically with the wavelength. In the equations shown, $m_{n}$ is the AWG diffraction order at wavelength $\lambda_{n}, f$ is the lens focal length, and $d$ is the grating period of the free-space demultiplexer.

The output-spot wavelengths are distributed in a raster fashion. The shortest wavelength signal is in the lower right, increasing in 50-GHz steps as you move up the column. There is a longer interval before you reach the next diffraction order, which starts at the next column to the left. The longest wavelength output is in the upper left corner. The design of the AWG (delay arm length distribution and output-waveguide arrangement) determines the space between diffraction orders.

Fig. 8 shows crosses at the measured position of maximum coupling efficiency for each channel into the fiber. These are plotted against their predicted positions represented by circles, calculated based on their diffraction order in the AWG and the grating equation, and measured focal length of the Fourier transform lens. The absolute position of the predicted and experimental data is aligned to minimize the RMS difference, which was $6.5 \mu \mathrm{m}$. In our measured data set, the column pitch decreases by more than half, from $0.268 \mathrm{~mm}$ near $1800 \mathrm{~nm}$ (at left) to $0.114 \mathrm{~mm}$ near $1160 \mathrm{~nm}$ (at right).

Irregularities in the output-spot position would be problematic if the output channels were to be coupled directly into

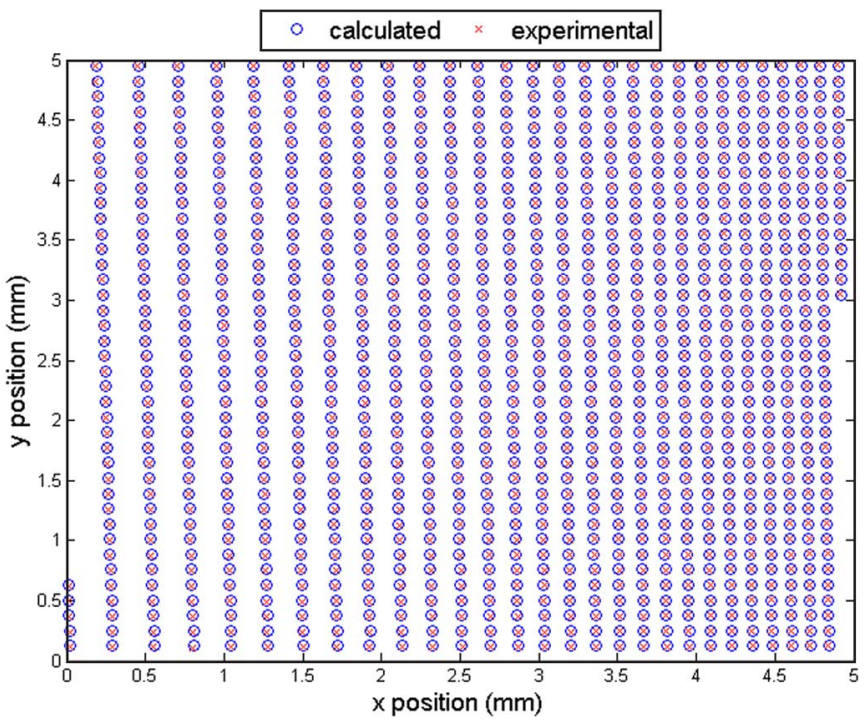

Fig. 8. Mapping of both theoretical and experimental spot locations in the output plane.

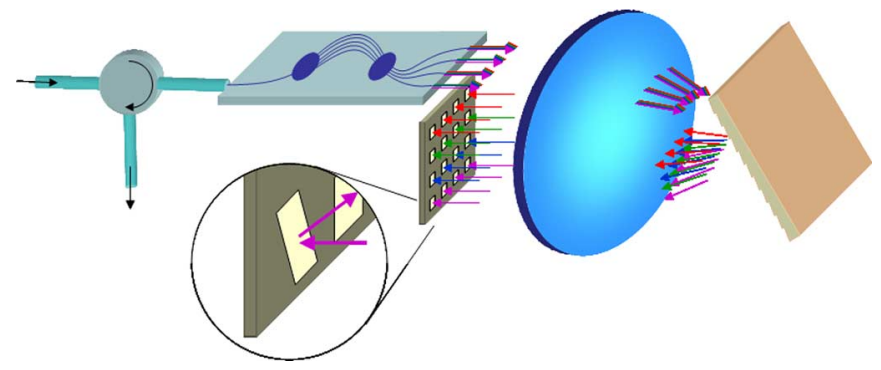

Fig. 9. Dynamic spectral equalizer employing the demultiplexer with an MEMS mirror array.

a 2-D fiber array: A lateral offset of $5 \mu \mathrm{m}$ would change the insertion loss by approximately $5 \mathrm{~dB}$. In the intended application, however, the signals are incident on optoelectronic detectors (for signal monitoring) or on microelectromechanical system (MEMS) mirrors or reflective liquid crystal modulators (for wavelength switching and equalization). The large lateral pitch between active regions makes it straightforward to fabricate individual devices with oversized apertures. In such devices, reflected signals are automatically realigned to the output fibers when they are remultiplexed by a second pass through the optical system [8], [9]. A dynamic spectral equalizer shown in Fig. 9 serves as an example of a typical setup where tiltable MEMS mirrors control individual channel alignment for attenuation control in a double pass configuration.

\section{OUtPut Channel Characterization}

The collected signals were relayed to an optical spectrum analyzer to measure the channel passband profiles shown in Figs. 10 and 11. Fig. 10 shows the insertion loss of a single channel at $1533.7 \mathrm{~nm}$ as well as its intermediate passband spectrum immediately following the AWG. The AWG insertion 


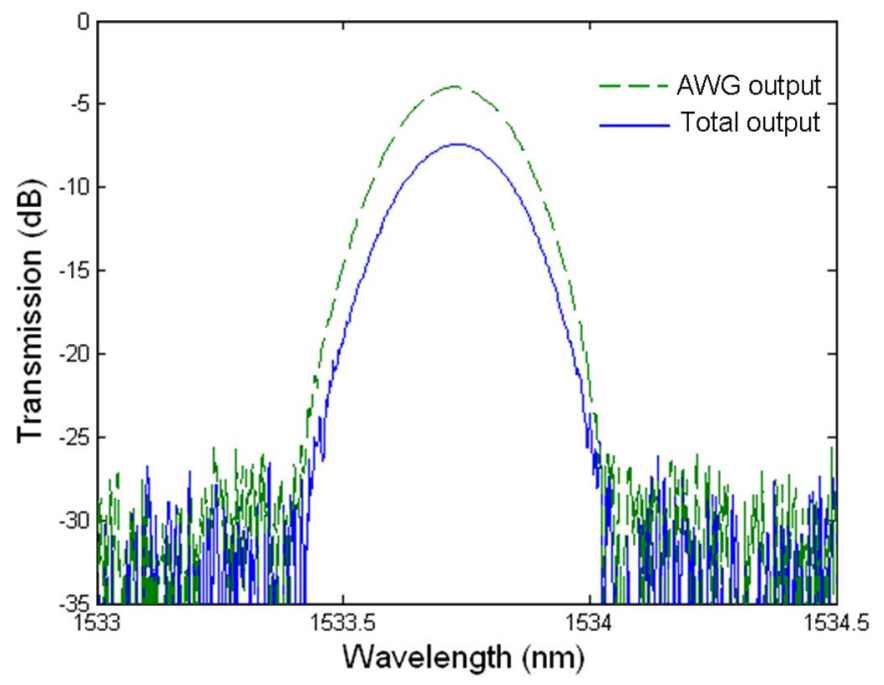

Fig. 10. Insertion loss and passband profile for a single output channel (solid line) closely following the Gaussian profile of the AWG output in the intermediate demultiplexing stage.

loss was $4 \mathrm{~dB}$, and a combined additional loss of $3.4 \mathrm{~dB}$ came from the grating demultiplexer, the $\mathrm{v}$-groove array, and the fiber connections. The Gaussian profile, the 0.24-nm 3-dB rolloff, and the 25-dB signal-to-background ratio of the AWG are maintained in the fully demultiplexed output.

In wavelength-division-multiplexing systems, crosstalk from neighboring channels is a concern. In our 2-D raster-positioned array of outputs, crosstalk may also come from adjacent channels in the array. This includes channels that are one FSR over in the neighboring diffraction orders. Fig. 12 shows the output spectrum of a single channel with the adjacent spots labeled where their passbands would appear. This reveals that there is no leakage of the signal into neighboring channels.

Fig. 11 shows the insertion loss of all 1092 channels, superimposed on a single $600-\mathrm{nm}$ spectrum. The plot is sectioned to fit into a column format. The increase in the background noise near the minimum $(1140 \mathrm{~nm})$ and maximum $(1750 \mathrm{~nm})$ wavelengths is due to the lower input signal levels and OSA sensitivity at these wavelengths. Insertion losses over the entire spectral range vary smoothly between 7 and $18 \mathrm{~dB}$ with the greatest losses occurring in channels below $1300 \mathrm{~nm}$. This is a result of the wavelength dependent losses of the two stages of demultiplexer at wavelengths far outside of their intended C-band function.

If we only accept channels with less than 10-dB insertion losses, our channel count is reduced to approximately 663 channels. With $80 \%$ bandwidth utilization, our demultiplexer would manage approximately $30 \%(26.5 \mathrm{THz})$ of the total spectral bandwidth. Much of the unaccounted data comes from high insertion losses far outside of the C-band wavelengths. Near the C-band, specifically at $1533.75 \mathrm{~nm}$, we measured 4-dB loss from the AWG and 3.4-dB loss from the FSG. At approximately $1730 \mathrm{~nm}$, both of the components exhibit almost no change in performance with $5-\mathrm{dB}$ loss from the AWG and 3-dB loss from the grating. We see the components start to fail further away from the C-band below $1300 \mathrm{~nm}$. At $1135 \mathrm{~nm}$, we measured 7-dB loss from the AWG and an
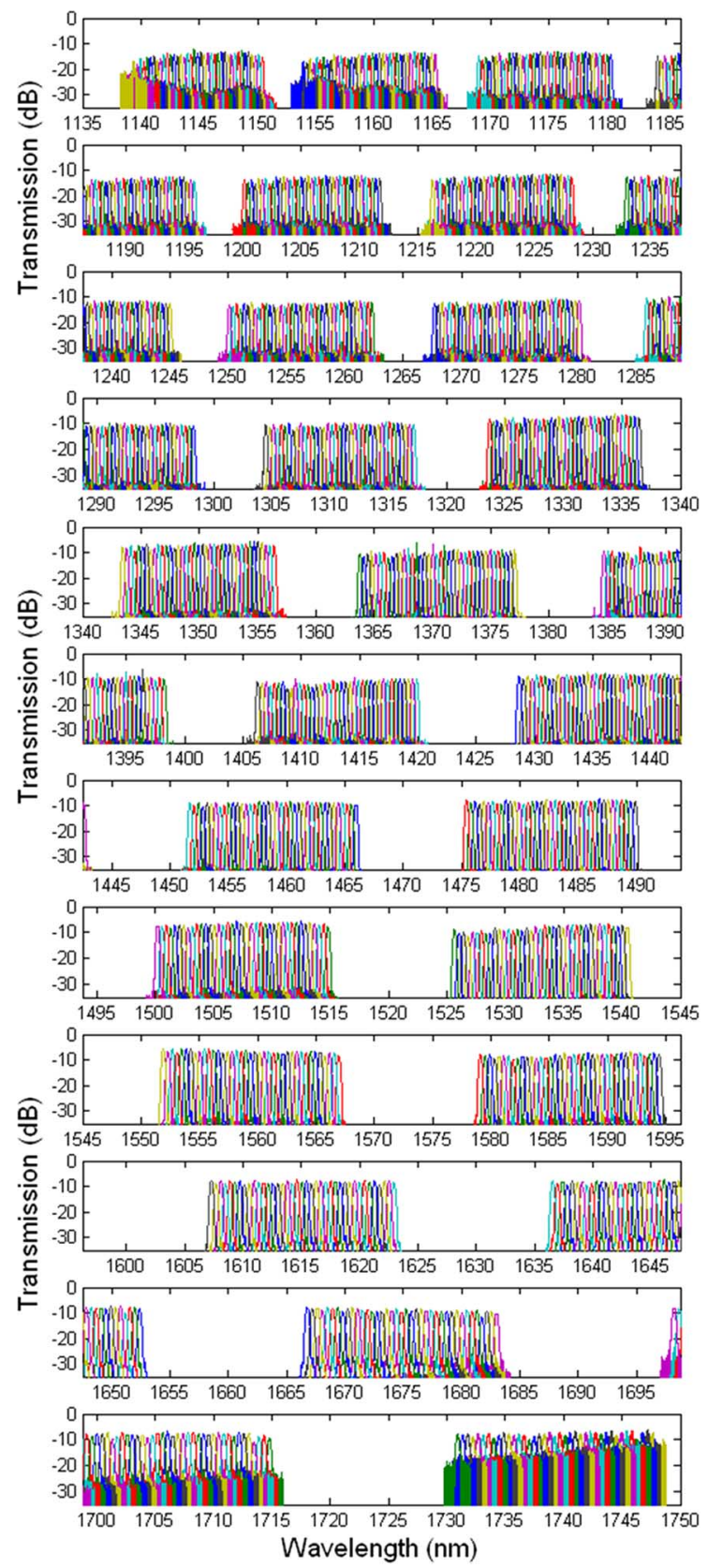

Fig. 11. All 1092 output channels superimposed over the full $600-\mathrm{nm}$ spectrum showing a smooth variation in insertion loss.

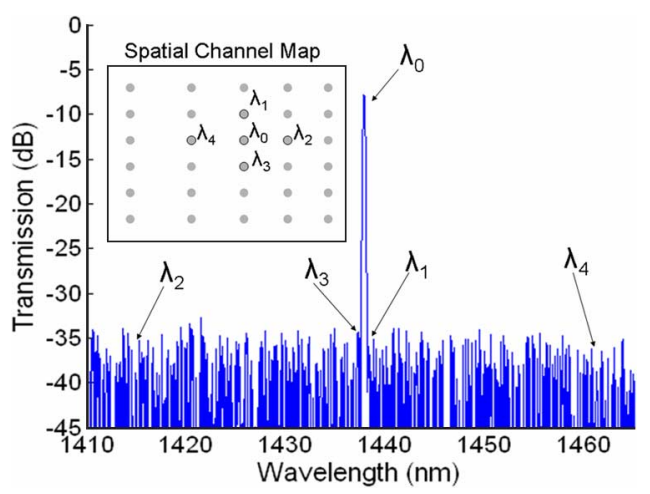

Fig. 12. Wide spectrum of a single channel showing no crosstalk between adjacent spots in the array. 


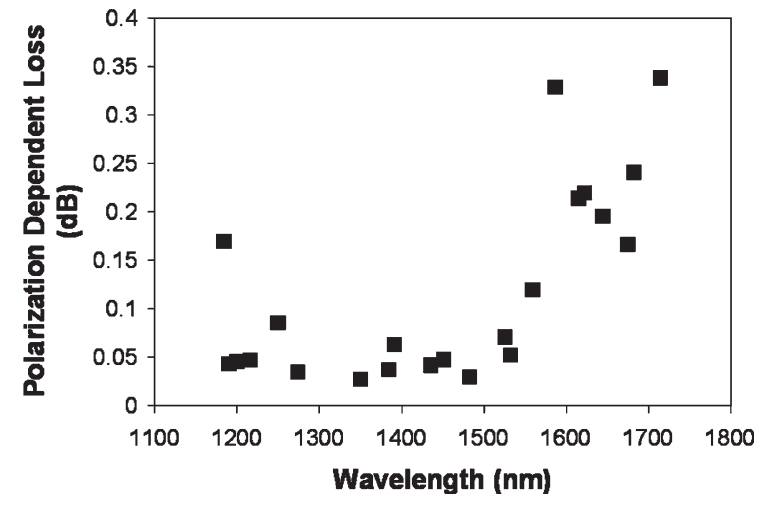

Fig. 13. Sampled PDL across the demultiplexer wavelength range.

even greater 10.5-dB loss from the FSG demultiplexer. These results show that the usable bandwidth in our setup is largely limited by the reflectivity of the grating demultiplexer and not by the AWG. Fig. 11 also shows that much of the spectrum is unused between diffraction orders. This is inherent from our AWG and can be avoided with a suitable AWG design. Customized AWG fabrication also allows flexibility in the channel spacings to fit a different number of total channels in the usable wavelength range. For example, the two-stage AWG demultiplexer developed by Nippon Telegraph and Telephone (NTT) Corp. generates 4200 channels over a shorter range by using an AWG with 5-GHz channel pitch [3]. The capacity of this demultiplexing scheme is only limited by the wavelengths that are filtered out through the components.

Both waveguide and free-space multiplexers can introduce significant polarization dependent loss (PDL). Current fabrication techniques have been proven effective in minimizing PDL in commercial AWG and are effective over a broad wavelength spectrum. PDL in planar diffraction gratings depends on operating spectrum and dispersion, but for the relatively course pitch, we require $(75 \mathrm{lp} / \mathrm{mm}$, as opposed to the $600 \mathrm{lp} / \mathrm{mm}$ used in [7]) low PDL gratings available: Ours had a specification of less than $0.1 \mathrm{~dB}$ over the bandwidth of our supercontinuum source. When the AWG and the grating are combined in the 2-D demultiplexing system, the total PDL across the active spectrum is less than $0.4 \mathrm{~dB}$. This is shown in Fig. 13 which reveals very little wavelength dependence on PDL.

Although the spectral passband profile of the AWG was maintained, it was possible that the second-stage demultiplexer introduced a nonuniform chromatic dispersion which could adversely impact data transmission. To verify that this was not the case, we performed BER tests to measure any loss in signal integrity. The output fiber was positioned to collect the 1543.9-nm wavelength channel. We then modulated a 1543.9-nm signal with a pseudorandom binary sequence and nonreturn-to-zero coding to perform BER tests on the demultiplexer. We used both 2.5- and 10-Gb/s modulation rates. Fig. 14 shows the results of these tests, and it is evident that the demultiplexer maintains signal quality. The penalty suffered by the $2.5-\mathrm{Gb} / \mathrm{s}$ signal was superimposed on the back-to-back signal, and the $10-\mathrm{Gb} / \mathrm{s}$ signal showed a still-negligible power penalty of $0.1 \mathrm{~dB}$. Since all channels encounter the same optical components, we expect similar results for the remaining channels.

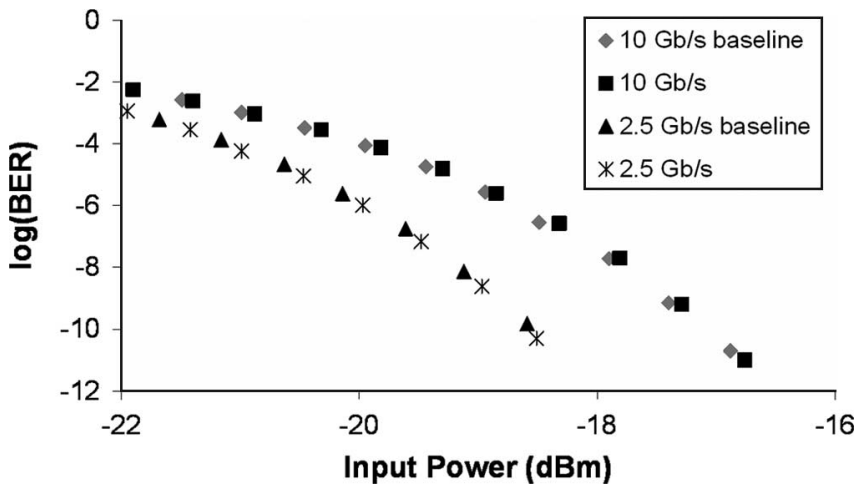

Fig. 14. BER testing of the 1543.9 channel of the demultiplexer showing a negligible penalty at both 2.5 and $10 \mathrm{~Gb} / \mathrm{s}$.

\section{CONCLUSION}

We have demonstrated a 2-D wavelength demultiplexer with 1092 channels spread over a 600-nm spectral band. If we assume an $80 \%$ utilization of the $50-\mathrm{GHz}$ pitch channels, this device supports a record aggregate potential data capacity of up to $44 \mathrm{~Tb} / \mathrm{s}$. The channel passbands were Gaussian in profile and showed to support $10-\mathrm{Gb} / \mathrm{s}$ data modulation without significant power penalty. The outputs were characterized using a single scanned fiber, but this demultiplexer is primarily intended to be used in wavelength switching, equalization, and monitoring components using MEMS, inductance-capacitance $(L-C)$, and optoelectronic devices. We believe that the total channel capacity of such array-parallel demultiplexers can be increased to 5000 or more channels by optimizing the AWG to reduce dead space between diffraction orders and by designing the FSG to operate over a 1200-nm spectral band.

\section{REFERENCES}

[1] P. P. Mitra and J. B. Stark, "Nonlinear limits to the information capacity of optical fibre communications," Nature, vol. 411, no. 6841, pp. 1027-1030, Jun. 2001.

[2] K. Fukuchi, T. Kasamatsu, M. Morie, R. Ohhira, T. Ito, K. Sekiya, D. Ogasahar, and T. Ono, "10.92-Tb/s $\left(273^{*} 40-\mathrm{Gb} / \mathrm{s}\right)$ tripleband/ultra-dense WDM optical-repeatered transmission experiment," in Proc. Opt. Fiber Commun. Conf. (IEEE Cat. 01CH37171), 2001, pp. PD24-1-PD24-3.

[3] K. Takada, M. Abe, T. Shibata, and K. Okamoto, "5 GHz-spaced 4200channel two-stage tandem demultiplexer for ultra-multi-wavelength light source using supercontinuum generation," Electron. Lett., vol. 38, no. 12, pp. 572-573, Jun. 2002.

[4] C. Dragone and J. E. Ford, "Free-space/arrayed waveguide router," U.S. Patent 6263 127, Jul. 17, 2001.

[5] A. M. Weiner and S. Xiao, "2-D wavelength demultiplexer with potential for $\geq 1000$ channels in the C-band," Opt. Express, vol. 12, no. 13, pp. 2895-2902, 2004.

[6] T. K. Chan, M. Abashin, and J. E. Ford, "2-D array wavelength demultiplexing by hybrid waveguide and free-space optics," presented at the OSA/IEEE Optical Fiber Commun. Conf., Anaheim CA, Mar. 2005, Paper OThV7.

[7] A. Dutta, N. Dutta, and M. Fujiwara, WDM Technologies: Passive Optical Components. San Diego, CA: Academic, 2003, p. 61.

[8] J. E. Ford, V. A. Aksyuk, D. J. Bishop, and J. A. Walker, "Wavelength add/drop switching using tilting micromirrors," J. Lightw. Technol., vol. 17, no. 5, pp. 904-911, May 1999.

[9] D. M. Marom, D. T. Neilson, D. S. Greywall, C.-S. Pai, N. R. Basavanhally, V. A. Aksyuk, D. O. Lopez, F. Pardo, M. E. Simon, Y. Low, P. Kolodner, and C. A. Bolle, "Wavelength-selective $1 \times K$ switches using free-space optics and MEMS micromirrors: Theory, design, and implementation," J. Lightw. Technol., vol. 23, no. 4, pp. 1620-1630, Apr. 2005. 
Trevor K. Chan was born in Edmonton, AB, Canada, on March 6, 1980. He received the B.Sc. degree in engineering physics from the University of Alberta, Edmonton, in 2002 and the M.Sc. degree in electrical and computer engineering from University of California, San Diego (UCSD), La Jolla, and 2004 where he is currently working toward the Ph.D. degree in electrical and computer engineering.

He was with the Photonics Systems Integration Laboratory, UCSD, in 2003. His research interests include free-space optical communications, optical modulators, and wavelength division multiplexing technologies.

Jason Karp received the B.S. degree in electrical engineering from the University of Miami, Miami, FL, in 2004. He is currently working toward the M.A. and Ph.D. degrees at the University of California, San Diego (UCSD), La Jolla.

He joined the Photonics Systems Integration Laboratory, UCSD, in 2005. His current research interests include free-space communication systems, imaging, and wavefront sensing.

Rui Jiang was born in Hubei, China, in 1978. He received the B.S. degree in engineering physics from Tsinghua University, Beijing, China, in 2000 and the M.A. degree in laser plasma physics and imaging science from University of Rochester, Rochester, NY, in 2004. He is currently working toward the Ph.D. degree at the Department of Electrical and Computer Engineering, University of California, San Diego (UCSD), La Jolla.

He was with the China Institute of Atomic Energy, working on laser plasma physics for two years. His interest lies in the nonlinear fiber optics, especially in parametric process.

Nikola Alic is currently working toward the Ph.D. degree at the University of California, San Diego, La Jolla.
Stojan Radic graduated from The Institute of Optics, University of Rochester, Rochester, NY

He has subsequently served as Senior Scientist with Corning Inc., Corning, NY, member of the Technical Staff at Bell Laboratories, and Nortel Networks Chair at Duke University, Durham, NC. He is currently an Associate Professor leading the Optical Networking Laboratory at the University of California, San Diego (UCSD), La Jolla/CalIT2. His research is focused on parametric processing and its use in all-optical networks.

Mr. Radic currently serves on the Conference on Lasers and Electro-Optics (CLEO), Optical Fiber Communications (OFC), and Optical Amplifiers and Their Applications (OAA) committees and is an Optical Communications Editor for Optics Express.

Christopher F. Marki was born in San Jose, CA, in August 1980. He received the B.S. degree in electrical engineering from Duke University, Durham, NC, in 2002. He subsequently matriculated to the University of California, San Diego (UCSD), La Jolla, where he received the M.S. degree in photonics in June 2004, where he is currently working toward the Ph.D. degree in optical communications.

He has held internships at Lucent Technologies and Anritsu Corporation and works closely with the Ziva Corporation, San Diego and Marki Microwave, Morgan Hill, CA.

Joseph E. Ford received the Ph.D. degree from University of California, San Diego (UCSD), La Jolla, in 1992 and the M.A. degrees in physics and optical engineering from the University of British Columbia, Vancouver, BC, Canada, and University of Rochester, Rochester, NY, respectively.

He is a coauthor of 35 refereed journal articles and 45 U.S. patents on optical components and systems. He was a member of Bell Labs' Advanced Photonics Research Department from 1994 to 2000, where he developed optoelectronics and micro-opto-mechanics for optical communications. From 2000 to 2002, he was with Optical Micromachines, San Diego, CA. In 2002, he became an Associate Professor with the Department of Electrical and Computer Engineering, USCD, where he leads the Photonic Systems Integration Laboratory. 